$$
v_{n}(x)=\frac{\Gamma[(1-x) n+1]}{\Gamma(1+n)}
$$

the Mittag-Leffler convergence factor:

$$
v_{n}(x)=\frac{1}{\Gamma(1+n x)} ;
$$

and the Dirichlet series convergence factors:

$$
v_{n}(x)=e^{-\lambda(n) x},
$$

where $\lambda(n)$ must be a logarithmico-exponential function of $n$ which tends to infinity with $n$ but not as slowly as $\log n$ nor faster than $n^{\Delta}$, where $\Delta$ is any constant however large.

NORTHWESTERN UNIVERSITY

\title{
A THEOREM ON SYMMETRIC DETERMINANTS
}

\author{
BY W. V. PARKER
}

1. Introduction. In a recent paper* the writer proved the following theorem.

If $D=\left|a_{i j}\right|$ is a real symmetric determinant of order $n, n>5$, in which $a_{i i}=0,(i=1,2, \cdots, n)$, and $M$ is any principal minor of $D$ of order $n-1$, then if all fourth order princifal minors of $M$ are zero, $D$ vanishes.

The purpose of the present note is to establish a second theorem of a similar nature which applies to complex as well as to real determinants. It will be shown also that when $a_{i j},(i \neq j)$, $(i, j=1,2, \cdots, n)$, is real and different from zero the conditions of this second theorem imply those of the above.

2. A Second Theorem. The theorem with which this note is concerned may be stated as follows.

TheOREM. If $D=\left|a_{i j}\right|$ is a symmetric determinant of order $n$, $n>5$, in which $a_{i i}=0,(i=1,2, \cdots, n)$, and $M$ is any principal minor of $D$ or order $n-1$, then if all fourth order principal minors of $D$, which are not minors of $M$, are zero, $D$ vanishes.

* This Bulletin, vol. 38 (1932), p. 259. 
There is no loss of generality in assuming that $M$ is the minor in the lower right hand corner of $D$ and we will make this assumption throughout. The theorem is trivial if all elements of the first row are zero. We will assume, therefore, that there is at least one element in the first row which is not zero and we may take this element to be $a_{12}$.

We will first show that the theorem is true for $n=6$ and $n=7$ and then show that the general theorem follows from these two special cases. For convenience we will write each element as the square of a number which may be either real or complex.

3. The Case $n=6$. For this case we may write

$$
D=\left|\begin{array}{llllll}
0 & \alpha^{2} & \beta^{2} & \gamma^{2} & \delta^{2} & \epsilon^{2} \\
\alpha^{2} & 0 & a^{2} & b^{2} & c^{2} & d^{2} \\
\beta^{2} & a^{2} & 0 & x^{2} & y^{2} & z^{2} \\
\gamma^{2} & b^{2} & x^{2} & 0 & u^{2} & v^{2} \\
\delta^{2} & c^{2} & y^{2} & u^{2} & 0 & w^{2} \\
\epsilon^{2} & d^{2} & z^{2} & v^{2} & w^{2} & 0
\end{array}\right|
$$

where $\alpha \neq 0$. Let us denote by $M_{i j}$ the fourth-order principal minor of the determinant $D$ obtained by striking out the $i$ th and $j$ th rows and the $i$ th and $j$ th columns. We then have, by hypothesis, $M_{i j}=0,(i=2,3,4,5),(j=3,4,5,6),(i<j)$. Since $M_{23}=(\gamma w+\delta v+\epsilon u)(\gamma u+\delta v-\epsilon u)(\gamma w-\delta v+\epsilon u)(\gamma w-\delta v-\epsilon u)=0$, either $\gamma^{2} w^{2}=(\epsilon u+\delta v)^{2}$ or $\gamma^{2} w^{2}=(\epsilon u-\delta v)^{2}$. Similar relations are obtained from the fact that the other minors are zero.

If now we subtract $\beta^{2} / \alpha^{2}$ times the second column from the third, $\gamma^{2} / \alpha^{2}$ times the second column from the fourth, $\delta^{2} / \alpha^{2}$ times the second column from the fifth, and $\epsilon^{2} / \alpha^{2}$ times the second column from the sixth, and then transform the rows in the same way, we get

$$
D=-\left(1 / \alpha^{4}\right)\left|\begin{array}{cccc}
-2 \beta^{2} a^{2} & \alpha^{2} x^{2}-\beta^{2} b^{2}-\gamma^{2} a^{2} & \alpha^{2} y^{2}-\delta^{2} a^{2}-\beta^{2} c^{2} & \alpha^{2} z^{2}-\beta^{2} d^{2}-\epsilon^{2} a^{2} \\
\alpha^{2} x^{2}-\beta^{2} b^{2}-\gamma^{2} a^{2} & -2 \gamma^{2} b^{2} & \alpha^{2} u^{2}-\gamma^{2} c^{2}-\delta^{2} b^{2} & \alpha^{2} v^{2}-\gamma^{2} d^{2}-\epsilon^{2} b^{2} \\
\alpha^{2} y^{2}-\delta^{2} a^{2}-\beta^{2} c^{2} & \alpha^{2} u^{2}-\gamma^{2} c^{2}-\delta^{2} b^{2} & -2 \delta^{2} c^{2} & \alpha^{2} w^{2}-\delta^{2} d^{2}-\epsilon^{2} c^{2} \\
\alpha^{2} z^{2}-\beta^{2} d^{2}-\epsilon^{2} a^{2} & \alpha^{2} v^{2}-\gamma^{2} d^{2}-\epsilon^{2} b^{2} & \alpha^{2} w^{2}-\delta^{2} d^{2}-\epsilon^{2} c^{2} & -2 \epsilon^{2} d^{2}
\end{array}\right| .
$$

Since $M_{34}=M_{35}=M_{36}=M_{45}=M_{46}=M_{56}=0$, if any one of the quantities $a, b, c, d, \beta, \gamma, \delta, \epsilon$ is zero, every element in the row and column of (2) containing this quantity will be zero and 
hence $D=0$. In a similar way we may show that when $M_{i j}=0$, $(i=2,3,4,5),(j=3,4,5,6),(i<j), D$ vanishes if any element not in the principal diagonal is zero.

Let us now consider the case when all elements outside of the principal diagonal are different from zero. The computation here is simplified somewhat by assuming $\alpha=\beta=\gamma=\delta=\epsilon=1$ and this may be done without loss of generality.*

Since $M_{34}=M_{35}=M_{36}=M_{45}=M_{46}=M_{56}=0$, we may write

$$
D=-16 a^{2} b^{2} c^{2} d^{2} \Delta,
$$

where

$$
\Delta=\left|\begin{array}{cccc}
-1 & \pm 1_{x} & \pm 1_{y} & \pm 1_{z} \\
\pm 1_{x} & -1 & \pm 1_{u} & \pm 1_{v} \\
\pm 1_{y} & \pm 1_{u} & -1 & \pm 1_{w} \\
\pm 1_{z} & \pm 1_{v} & \pm 1_{w} & -1
\end{array}\right|
$$

where $\pm 1_{x}$ is +1 or -1 according as, in $M_{56}$,

$$
x^{2}=(a+b)^{2} \quad \text { or } \quad x^{2}=(a-b)^{2} \text {, }
$$

and similarly for the others.

Since $a, b, c, d$ are all different from zero, $D$ vanishes if and only if $\Delta$ vanishes. Since $M_{45}=M_{46}=M_{56}=0$, one of the following must be true:

\begin{tabular}{c|c|c|c|c|c|c|c|c|} 
& 1 & 2 & 3 & 4 & 5 & 6 & 7 & 8 \\
\hline$x$ & + & - & + & + & + & - & - & - \\
\hline$y$ & + & + & - & + & - & + & - & - \\
\hline$z$ & + & + & + & - & - & - & + & - \\
\hline
\end{tabular}

$$
\begin{aligned}
& * D=\alpha^{4} \beta^{4} \gamma^{4} \delta^{4} \epsilon^{4} D_{1} \text {, where } \\
& D_{1}=\left|\begin{array}{cccccc}
0 & 1 & 1 & 1 & 1 & 1 \\
1 & 0 & a^{2} /\left(\alpha^{2} \beta^{2}\right) & b^{2} /\left(\alpha^{2} \gamma^{2}\right) & c^{2} /\left(\alpha^{2} \delta^{2}\right) & d^{2} /\left(\alpha^{2} \epsilon^{2}\right) \\
1 & a^{2} /\left(\alpha^{2} \beta^{2}\right) & 0 & x^{2} /\left(\beta^{2} \gamma^{2}\right) & y^{2} /\left(\beta^{2} \delta^{2}\right) & z^{2} /\left(\beta^{2} \epsilon^{2}\right) \\
1 & b^{2} /\left(\alpha^{2} \gamma^{2}\right) & x^{2} /\left(\beta^{2} \gamma^{2}\right) & 0 & u^{2} /\left(\gamma^{2} \delta^{2}\right) & v^{2} /\left(\gamma^{2} \epsilon^{2}\right) \\
1 & c^{2} /\left(\alpha^{2} \delta^{2}\right) & y^{2} /\left(\beta^{2} \delta^{2}\right) & u^{2} /\left(\gamma^{2} \delta^{2}\right) & 0 & w^{2} /\left(\delta^{2} \epsilon^{2}\right) \\
1 & d^{2} /\left(\alpha^{2} \epsilon^{2}\right) & z^{2} /\left(\beta^{2} \epsilon^{2}\right) & v^{2} /\left(\gamma^{2} \epsilon^{2}\right) & w^{2} /\left(\delta^{2} \epsilon^{2}\right) & 0
\end{array}\right| .
\end{aligned}
$$

Since $\alpha, \beta, \gamma, \delta, \epsilon$ are all different from zero, $D$ and $D_{1}$ vanish simultaneously and so do their corresponding minors. 
where 1 means $x^{2}=(a+b)^{2}, y^{2}=(a+c)^{2}, z^{2}=(a+d)^{2}$, and similarly for the others.

Since $M_{34}=M_{35}=M_{36}=0$, one of the following must be true:

\begin{tabular}{c|c|c|c|c|c|c|c|c|} 
& $1^{\prime}$ & $2^{\prime}$ & $3^{\prime}$ & $4^{\prime}$ & $5^{\prime}$ & $6^{\prime}$ & $7^{\prime}$ & $8^{\prime}$ \\
\hline$u$ & + & - & + & + & + & - & - & - \\
\hline$v$ & + & + & - & + & - & + & - & - \\
\hline$w$ & + & + & + & - & - & - & + & - \\
\hline
\end{tabular}

where $1^{\prime}$ means $u^{2}=(b+c)^{2}, v^{2}=(b+d)^{2}, w^{2}=(c+d)^{2}$, and similarly for the others. In substituting these values, it is sufficient to take only positive square roots since $D$ and all minors are even functions of their arguments.

If now we indicate the fact that $i$ and $j^{\prime}$ are both true by $\left(i, j^{\prime}\right)$, we find that $M_{23}=M_{24}=M_{25}=M_{26}=0$ is impossible for all cases except $\left(1,8^{\prime}\right) ;\left(2,4^{\prime}\right) ;\left(3,3^{\prime}\right) ;\left(4,2^{\prime}\right) ;\left(5,4^{\prime}\right) ;\left(6,3^{\prime}\right)$; $\left(7,2^{\prime}\right) ;\left(8,8^{\prime}\right)$. In each of these cases $\Delta$ is zero and hence $D$ vanishes. The theorem is true, therefore, for $n=6$.

4. The Case $n=7$. In this case we write

$$
D=\left|\begin{array}{lllllll}
0 & \alpha^{2} & \beta^{2} & \gamma^{2} & \lambda^{2} & \mu^{2} & \nu^{2} \\
\alpha^{2} & 0 & a^{2} & b^{2} & c^{2} & d^{2} & f^{2} \\
\beta^{2} & a^{2} & 0 & m^{2} & n^{2} & p^{2} & q^{2} \\
\gamma^{2} & b^{2} & m^{2} & 0 & x^{2} & y^{2} & z^{2} \\
\lambda^{2} & c^{2} & n^{2} & x^{2} & 0 & u^{2} & v^{2} \\
\mu^{2} & d^{2} & p^{2} & y^{2} & u^{2} & 0 & w^{2} \\
\nu^{2} & f^{2} & q^{2} & z^{2} & v^{2} & w^{2} & 0
\end{array}\right|,
$$

where $\alpha \neq 0$. Denote by $M_{i j k}$ the fourth order principal minor of $D$ obtained by striking out the $i$ th, $j$ th, and $k$ th rows and columns. Then by hypothesis $M_{i j k}=0,(i=2,3,4,5),(j=3,4,5,6)$, $(k=4,5,6,7),(i<j<k)$. Each of these minors may be written as the product of four factors as in the previous case.

By a transformation similar to the one above and making use of the fact that $M_{i j k}=0,(i=3,4,5),(j=4,5,6),(k=5,6,7)$, $(i<j<k)$, we find that $D$ is zero if any element, outside the principal diagonal, in the first two rows is zero. Similarly we may 
show that when $M_{i j k}=0,(i=2,3,4,5),(j=3,4,5,6),(k=4,5$, $6,7),(i<j<k), D$ vanishes if any element outside the principal diagonal is zero.

Let us suppose now that all elements outside of the principal diagonal are different from zero. As before we may assume $\alpha=\beta=\gamma=\lambda=\mu=\nu=1$ and we get

$$
D=-32 a^{2} b^{2} c^{2} d^{2} f^{2} \Delta,
$$

where

$$
\Delta=\left|\begin{array}{ccccc}
-1 & \pm 1_{m} & \pm 1_{n} & \pm 1_{p} & \pm 1_{q} \\
\pm 1_{m} & -1 & \pm 1_{x} & \pm 1_{y} & \pm 1_{z} \\
\pm 1_{n} & \pm 1_{x} & -1 & \pm 1_{u} & \pm 1_{v} \\
\pm 1_{p} & \pm 1_{y} & \pm 1_{u} & -1 & \pm 1_{w} \\
\pm 1_{q} & \pm 1_{z} & \pm 1_{v} & \pm 1_{w} & -1
\end{array}\right|
$$

where $\pm 1_{m}$ is +1 or -1 according as, in $M_{567}, m^{2}=(a+b)^{2}$ or $m^{2}=(a-b)^{2}$, and similarly for the others.

Since $a, b, c, d, f$ are all different from zero, $D$ vanishes if and only if $\Delta$ vanishes. Since $M_{356}=M_{357}=M_{367}=0$, we must have one of the cases under (I) above, where by 1 in (I) we now mean $x^{2}=(b+c)^{2}, y^{2}=(b+d)^{2}, z^{2}=(b+f)^{2}$ and similarly for the others. Since $M_{345}=M_{346}=M_{347}=0$, we must have one of the cases under (II) above, where by $1^{\prime}$ in (II) we now mean $u^{2}=(c+d)^{2}, v^{2}$ $=(c+f)^{2}, w^{2}=(d+f)^{2}$, and similarly for the others.

As before, we will indicate the fact that $i$ and $j^{\prime}$ are both true by $\left(i, j^{\prime}\right)$. We find that $M_{234}=M_{235}=M_{236}=M_{237}=0$ is impossible for every case except $\left(1,8^{\prime}\right) ;\left(2,4^{\prime}\right) ;\left(3,3^{\prime}\right) ;\left(4,2^{\prime}\right) ;\left(5,4^{\prime}\right)$; $\left(6,3^{\prime}\right) ;\left(7,2^{\prime}\right) ;\left(8,8^{\prime}\right)$, and in each of these cases $\Delta$ is zero and hence $D$ vanishes. The theorem is true, therefore, for $n=7$.

The theorem for the general case now follows immediately. If there is an element in the first row which is not zero, there is a second order principal minor, not a minor of $M$, which is not zero. If now all third order principal minors which are not minors of $M$ are zero, $D$ is of rank two.* If there is a third order principal minor, not a minor of $M$, which is not zero, $D$ is of rank three provided that all fifth order principal minors which are not minors of $M$ are zero. If there is a fifth order principal

* Bôcher, Introduction to Higher Algebra, p. 57, Theorem 1. 
minor, not a minor of $M$, which is not zero, it follows from the two special cases above that $D$ is of rank five.* If, therefore, there is an element in the first row which is not zero, $D$ is of rank five or less.

If all elements outside of the principal diagonal are different from zero, then all fifth order principal minors of $D$, which are not minors of $M$, are zero and hence $D$ is of rank three. It will be sufficient to show that the fifth order minor, $K$, in the upper left hand corner of (1) is zero when the elements outside of the principal diagonal are all different from zero. $K$ is zero if and only if the third order minor, $\Delta^{\prime}$, in the upper left of (4) is zero. But $\Delta^{\prime}=2\left[1+\left( \pm 1_{x}\right)\left( \pm 1_{y}\right)\left( \pm 1_{u}\right)\right]$ and hence is zero in each of the cases, $\left(1,8^{\prime}\right) ;\left(2,4^{\prime}\right) ;\left(3,3^{\prime}\right) ;\left(4,2^{\prime}\right) ;\left(5,4^{\prime}\right) ;\left(6,3^{\prime}\right) ;\left(7,2^{\prime}\right)$; $\left(8,8^{\prime}\right)$, for which $M_{23}=M_{24}=M_{25}=M_{26}=0$ is possible.

That this may not be true when there are two zeros in the same rows is shown by the following example. Let

$$
D=\left|\begin{array}{cccccc}
0 & 1 & 1 & 1 & 1 & 0 \\
1 & 0 & a^{2} & (a+b)^{2} & b^{2} & c^{2} \\
1 & a^{2} & 0 & b^{2} & (a+b)^{2} & c^{2} \\
1 & (a+b)^{2} & b^{2} & 0 & a^{2} & c^{2} \\
1 & b^{2} & (a+b)^{2} & a^{2} & 0 & c^{2} \\
0 & c^{2} & c^{2} & c^{2} & c^{2} & 0
\end{array}\right|
$$

where $a, b$, and $a+b$ are all real and different from zero. All fourth order principal minors of $D$ except those that are minors of the fifth order minor in the lower right, are zero. The fifth order minor in the upper left hand corner of $D$ is not zero, however. $\dagger$

Mississippi Woman's College

* Bôcher, loc. cit.

† L. M. Blumenthal, this Bulletin, vol. 37 (1931), p. 755. 